\title{
Reduction of Single-Neuron Firing Uncertainty by Cortical Ensembles during Motor Skill Learning
}

\author{
Dana Cohen ${ }^{1}$ and Miguel A. L. Nicolelis ${ }^{1,2,3,4}$ \\ ${ }^{1}$ Department of Neurobiology and ${ }^{2}$ Center for Neuroengineering, Duke University Medical Center, Durham, North Carolina 27710, ${ }^{3}$ Department of \\ Biomedical Engineering, Duke University, Durham, North Carolina 27708, and ${ }^{4}$ Department of Psychological and Brain Sciences, Duke University, \\ Durham, North Carolina 27710
}

\begin{abstract}
Motor skill learning is usually characterized by shortening of response time and performance of faster, more stereotypical movements. However, little is known about the changes in neural activity that underlie these behavioral changes. Here we used chronically implanted electrode arrays to record neuronal activity in the rat primary motor cortex (MI) as animals learned to execute movements in two directions. Strong modulation of MI single-neuron activity was observed while movement duration of the animal decreased. Despite many learning-induced changes, the precision with which single neurons fire did not improve with learning. Hence, prediction of movement direction from single neurons was bounded. In contrast, prediction of movement direction using neuronal ensembles improved significantly with learning, suggesting that, with practice, neuronal ensembles learn to overcome the uncertainty introduced by single-neuron stochastic activity.
\end{abstract}

Key words: extracellular; motor cortex; movement; motion; motor activity; chronic recording; skill learning; neural variability

\section{Introduction}

A variety of changes in the primary motor cortex (MI) have been described during different types of motor learning, such as formation of associations between sensory cues and movements (Mitz et al., 1991; Germain and Lamarre, 1993; Laubach et al., 2000; Paz et al., 2003), adjustment to a novel environment (Shadmehr and Holcomb, 1997; Gandolfo et al., 2000; Li et al., 2001), and learning of a new motor skill (Kleim et al., 1998; Muellbacher et al., 2001; Ungerleider et al., 2002; Lotze et al., 2003).

Motor skill learning involves shortening of the animal's response time (preparatory period) and the ability to execute fast, highly stereotypical movements (Wolpert et al., 2001). So far, motor skill learning has been correlated with an increase in the number of synapses per neuron in layer V of MI (Kleim et al., 2002), increased levels of long-term potentiation in layers 2 and 3 (Rioult-Pedotti et al., 2000), increased excitability of MI (Bonato et al., 2002; Lotze et al., 2003), modification in the activated area before and after learning (Remple et al., 2001; Ungerleider et al., 2002), and long-term changes in the preferred direction of neurons during adaptation to alterations in an external force field (Gandolfo et al., 2000; Li et al., 2001). In addition, disruption of MI function immediately after learning prevents the consolidation of motor memories (Muellbacher et al., 2002). Despite this plethora of findings, the functional role of changes in MI neuronal activity during learning remains elusive.

Received Dec. 4, 2003; revised Feb. 23, 2004; accepted Feb. 25, 2004.

This work was supported by the Human Frontier Science Program (D.C.) and National Institutes of Health Grant DE13810 (M.A.L.N.).

Correspondence should be addressed to Dana Cohen, Department of Neurobiology, Duke University Medical Center, Box 3209, Durham, NC 27710. E-mail: danac@neuro.duke.edu.

D01:10.1523/JNEUROSCI.5361-03.2004

Copyright $\odot 2004$ Society for Neuroscience $\quad$ 0270-6474/04/243574-09\$15.00/0
To address this important issue, chronic neural ensemble recordings in the rat MI cortex were performed as rats learned a task that required them to move to one of two end points after auditory cues. The classical view of the motor cortex suggests that during the learning of these two movements, the firing rate of participating MI neurons should increase (Reina et al., 2001), and directional tuning should improve (Georgopoulos et al., 1986). On the basis of studies in perceptual learning, we considered two ways in which this increased specificity could be achieved: (1) increased discriminability, measured as the distance between the means of the firing rate distributions (Dayan and Abbott, 2001) of left or right movements (Gold et al., 1999); and (2) increased precision of neuronal firing, measured as a decrease in the variance of the firing rate distributions (Poggio et al., 1992). These possibilities are not exclusive, and both could occur simultaneously (Dosher and Lu, 1998). Using single-trial analyses, we show that although single-neuron activity was strongly modulated during motor skill learning, the firing rate of the MI ensemble did not increase. The increase in single-neuron modulation contributed to the enhancement in discriminability of singleneuron activity during leftward and rightward movements. However, the precision with which single neurons fire did not improve with learning and depended linearly on its average firing rate. To assess the firing variability of the population, we developed an algorithm that, on the basis of the statistical similarity of neural activity over different trials, predicts movement direction (Dubnov et al., 2002). As expected, the best prediction of movement direction by single neurons was limited by the variance of their firing and did not improve with learning. In contrast, prediction of movement direction using the neuronal ensemble improved significantly with learning. On the basis of these results, we propose that improvement in movement performance is achieved by 
collective neuronal ensemble activity, which overcomes the lack of reliability of single-neuron firing.

\section{Materials and Methods}

Behavioral paradigm. Initially, rats were trained to nose poke a hole and wait for a tone of $14 \mathrm{kHz}$ to receive $50 \mu \mathrm{l}$ of a water reward. The time delay to the tone was gradually increased until the rats consistently waited for $550-700 \mathrm{msec}$ during $85 \%$ of the trials. Rats were then removed from the water deprivation protocol and underwent surgery. After 7-10 d of recovery, the second part of training began. Rats waited for the cue as before; however, now either a high $(20 \mathrm{kHz})$ or a low $(8 \mathrm{kHz})$ tone was played. Depending on the tone played, the rats had to move toward one of two holes located either to the left $(20 \mathrm{kHz})$ or to the right $(8 \mathrm{kHz})$ of the center hole. If they performed the correct movement, they received 50 $\mu l$ of water as a reward. An error trial occurred when the rats nose poked the wrong side hole. In this case, rats were forced to repeat a trial until they performed it correctly to prevent the development of a side preference. The intertrial interval changed randomly, ranging between 15 and $30 \mathrm{sec}$.

An infrared source detector system was installed behind each door, allowing the detection of the times at which rats entered or retracted their snouts from a hole. Head retraction of more than few millimeters was detected by the infrared system, and the trial was canceled. The holes were shaped like a cone with a base of approximately the size of the rat's snout, which prevented the rats from turning their heads left or right inside the hole. To ensure capturing the entire movement, $50 \mathrm{msec}$ was subtracted from the time the infrared system detected the rat's head retraction and was added to the times at which rats nose poked a hole.

Surgery. All procedures were done in accordance with National Institutes of Health guidelines. The surgical procedure has already been described in detail (Nicolelis et al., 1997). In this study, two craniotomies were made bilaterally above the neck, back, and shoulder representation areas in MI, which are centered at $1 \mathrm{~mm}$ frontolateral to bregma (Remple et al., 2001). An array containing 16 Isonel-coated tungsten microwires arranged in a $4 \times 4$ matrix (custom design) was implanted in layer 5 of the cortex in each craniotomy.

Data collection. Single cells and multiunit activity were recorded simultaneously using a multichannel acquisition processor system (Plexon Inc, TX). The activity was initially sorted using an on-line sorting algorithm (Plexon Inc.). Only cells that had a clearly identified waveform with a signal-to-noise ratio of at least 3:1, measured on the oscilloscope, were used. At the end of the recording, cells were resorted using an off-line sorting algorithm (Plexon Inc.) to further confirm the quality of the recorded cells (Nicolelis et al., 2003). Single-unit analysis and multiunit analysis were conducted separately. However, because there was no substantial difference in the results in single- and multi-unit data, the data were combined for the purpose of presentation. Comparison of the waveforms, interspike intervals, and firing activity of the cells during the task suggests that $37 \%$ of the cells were recorded during more than one session. However, in the case of our analyses, it is more conservative to assume that the neurons recorded each day were different, so we used this assumption in our analysis. The neuronal ensemble consists of all the neurons recorded simultaneously in one animal during one session.

Data analyses. Single neurons were considered task-related if they significantly changed their firing rate during the preparatory period or during movement relative to their activity during the waiting period.

Comparison of firing frequency. A normalized firing rate difference function, $n f d=\left(f_{2}-f_{1}\right) /\left(f_{2}+f_{1}\right)$, was used to evaluate the changes in firing rate within a session; $f_{1}$ and $f_{2}$ are the average movement firing rates calculated during the first 30 trials performed in each direction (the early phase) and the last 30 trials performed in the same direction (the late phase), respectively. Values at $\sim 0$ reflect stable activity within a session, and values closer to 1 or -1 reflect an increase or decrease in firing rate at the end of a session, respectively.

Classification of single-trial outcome. The algorithm is a nonparametric method based on pair-wise distance; each trial is represented by its relation to all the other trials, and this relation (distance) is reestimated using a statistically motivated proximity measure. Specifically, the algorithm weighs the difference in activity over trials, assuming Poisson firing
(Averbeck and Lee, 2003) of individual neurons. The probability that a neuron fired $v$ spikes while its average firing rate was $r$, assuming a Poisson distribution, is:

$$
\text { (1) } p(v \mid r)=\frac{e^{-r} r^{-v}}{v !}
$$

The rate vector that is most likely to yield given spike counts on two independent trials $\left(v_{i}\right.$ and $\left.v_{j}\right)$ is the average of the two spike counts:

$$
\text { (2) } p\left(v_{\mathrm{i}}, v_{\mathrm{j}} \mid r\right)=p\left(v_{\mathrm{i}} \mid\left(v_{\mathrm{i}}+v_{\mathrm{j}}\right) / 2\right) \cdot p\left(v_{\mathrm{j}} \mid\left(v_{\mathrm{i}}+v_{\mathrm{j}}\right) / 2\right) .
$$

The similarity between two trials, $d_{\mathrm{ij}}$, is taken as the log probability that the corresponding spike count vectors were generated independently by the same maximum likelihood rate vector and calculated for all the neurons together. $d_{\mathrm{ij}}$ is called the similarity matrix:

$$
\begin{aligned}
d_{\mathrm{ij}}=\log \left(\prod_{\{n\}} p\left(v_{\mathrm{n}, \mathrm{i}} \mid r_{\mathrm{n}, \mathrm{ij}}\right) \cdot p\left(v_{\mathrm{n}, \mathrm{j}} \mid r_{\mathrm{n}, \mathrm{ij}}\right)\right)=\sum_{\{n\}}(\log ( & \left.p\left(v_{\mathrm{n}, \mathrm{i}} \mid r_{\mathrm{n}, \mathrm{ij}}\right)\right) \\
& \left.+\log \left(p\left(v_{\mathrm{n}, \mathrm{j}} \mid r_{\mathrm{n}, \mathrm{ij}}\right)\right)\right) .
\end{aligned}
$$

After calculating the similarity matrix, we used the "clustering according to friends" algorithm to separate the trials into two groups (Dubnov et al., 2002). After classification of all trials into two groups, we measured the percent overlap between the classified groups and the movement direction of each trial. An overlap of 100\% means that the two groups created by the algorithm matched completely the trial separation according to movement direction. For example, when the neuronal activity during movement to both sides is the same, the algorithm will successfully predict $\sim 50 \%$ of the trials because the trials are statistically indistinguishable. When there is no overlap in the distribution of firing rates to the two directions, the algorithm will successfully predict $100 \%$ of the trials. The algorithm can work equally well on data from single neurons and from multiple simultaneously recorded neurons. Each neuron is then treated as an independent variable with a Poisson distribution of its firing rate.

\section{Results}

\section{Behavioral characteristics}

Thirteen adult rats $(n=13)$ learned to perform the two-choice auditory conditional task (Fig. $1 A$; see Materials and Methods). On average, performance of animals reached criteria ( $80 \%$ correct trials) after $7 \mathrm{~d}$ (Fig. $1 \mathrm{~B}$ ). The average reaction time and movement duration significantly decreased from $395 \pm 48$ and $640 \pm 26 \mathrm{msec}$, respectively, on day 1 to $241 \pm 22$ and $338 \pm 21$ msec on day 5 (Fig. 1C; ANOVA, $p<0.01$; $p<0.0000001$, respectively). Reaction time decreased gradually and reached significance on the fifth day, whereas movement duration decreased significantly by the second day and plateaued (post hoc analysis). A more detailed analysis shows that movement duration and variability decreased significantly from the initial 30 trials (early trials) to the last 30 trials (late trials) of the first day (Fig. $1 D$; early trials, $876 \pm 56 \mathrm{msec}$; late trials, $459 \pm 23 \mathrm{msec}$; ANOVA, $p<$ 0.00001; Fig. $1 E$; early trials, $107 \pm 21 \mathrm{msec}$; late trials, $26 \pm 6$ msec; ANOVA, $p<0.00001)$. Movement duration decreased further between the first and second sessions (Fig. $1 D$; first day, late trials, $459 \pm 23 \mathrm{msec}$; second day, early trials, $385 \pm 10 \mathrm{msec}$; ANOVA, $p<0.01)$.

A two-choice conditional task is composed of two types of learning: motor skill learning (i.e., improved movement execution) and associative learning (i.e., creating an association from sensory cues to specific movements). Motor skill learning is reflected in a reduction of reaction time, movement duration, and movement variability. Associative learning entails a reduction in reaction time and an increase in the percent of correct trials. Here, movement duration and variability decreased mainly during the first day, whereas on the second day, the percent of correct 

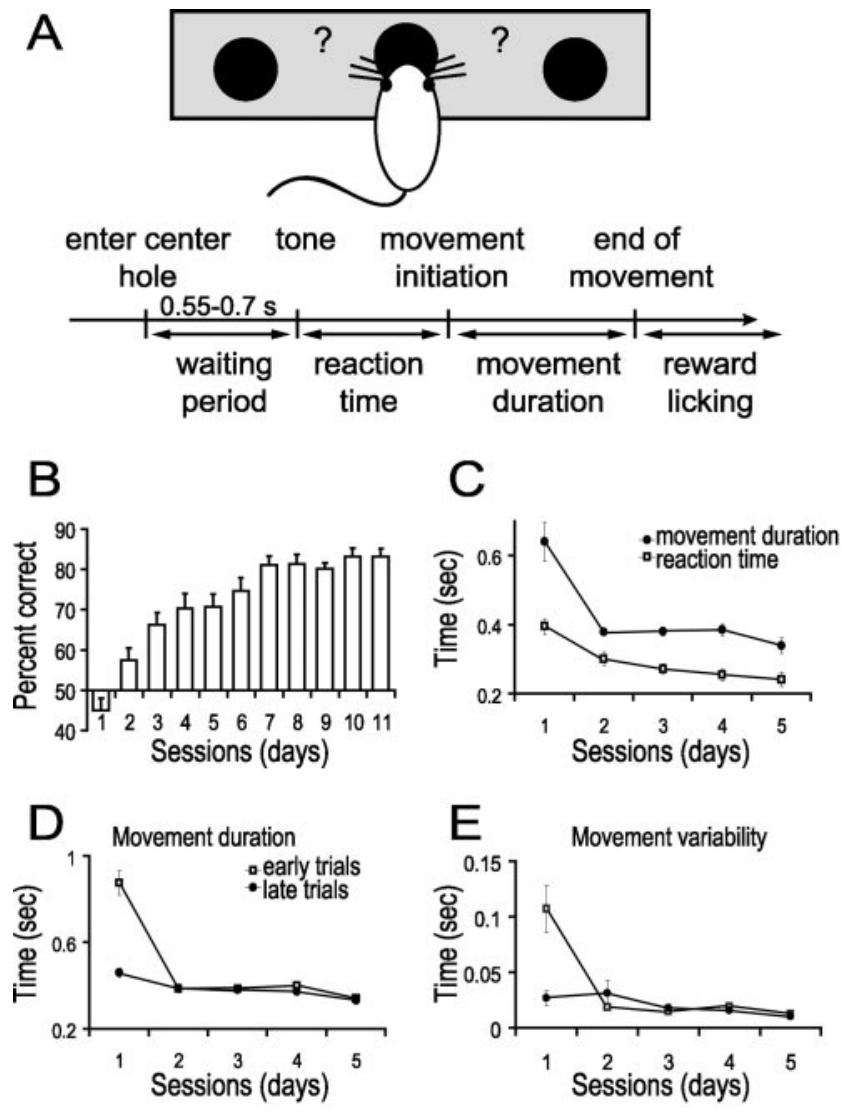

Figure 1. Practice decreased reaction times, movement times, movement variability, and error frequency. $A$, Schematic of the behavioral paradigm and time course of a trial. Filled circles represent holes that the rat can nose poke. The rat must discriminate a high tone from a low tone played while in the center hole and then move to the side hole specified by that tone to receive reward. $B, 0$ naverage, rats $(n=13)$ reached a performance level of $80 \%$ by the seventh day. $C$, Movement time (filled circles) and reaction time (open squares) significantly decreased with training ( $n=6$ in all analysis from here on). D, A significant reduction in movement duration occurred by the late trials (filled circles) of the first day and on the early trials (open squares) of the second day. E, A significant reduction in movement variability occurred from the early trials (open squares) to the late trials (filled circles) of the first day.

trials was still near chance (57\%). These data suggest that motor skill learning dominated the first day, whereas the associative learning took a longer period to occur. Therefore, we assume that measures of neural activity that change significantly during the first day and do not change on following days reflect motor skill learning.

\section{Learning-induced changes in neuronal activity}

Chronic and simultaneous recordings of neural activity from the neck, back, and shoulder representation areas in MI (Remple et al., 2001) were obtained in six male rats during the first three sessions of training. The numbers of single neurons recorded during sessions $1-3$ were 86,107 , and 101, respectively (average, $16.5 \pm 2.3$ per animal). Most of the recorded neurons were movement-related ( $87 \pm 3 \%$ ), whereas the number of neurons that responded during the preparatory period was smaller $(44 \pm$ $4 \%$; paired $t$ test, $p<0.01)$. The percent of task-related cells did not change significantly over days.

Because the most dramatic change in movement duration occurred on day 1 (Fig. 1D), we first compared the neural activity during the early and late trials of that day. MI neurons exhibited a variety of firing patterns during execution of the task. Qualita- tively, four classes of changes in the postevent time histograms (PETHs) dominated the spectrum of firing changes observed in our neuronal sample: development of firing modulation related to the task (Fig. $2 A ; 19 \%$ of the task-related neurons); strengthening of an already existing firing pattern (Fig. $2 B$; 24\%); enhancement or development of a decrease in firing (Fig. 2C;29\%); and, finally, shortening of the duration of movement-related activity (Fig. 2D;32\%). Some cells exhibited changes in neural activity from more than one of these types. In addition, the observed changes could occur selectively during movement to one side or during movement in both directions (Fig. 2, compare left, right). Qualitatively, neuronal firing patterns changed mainly during the first day, matching the time course of the changes in the behavior of animals. On the second and third days, most neurons exhibited steady and well-defined firing patterns (Fig. 3). We therefore focused on comparison of the neuronal activity during the early and late trials of day 1 and contrasted that with comparison of neuronal activity in the early and late trials of subsequent days.

For each trial, regardless of movement direction, we calculated 2 parameters: (1) the firing rate during movement and (2) whether a significant change in firing rate occurred within a small time window around movement initiation. The first parameter characterizes the neural correlates of movement, and the second parameter measures the strength of the instantaneous change in firing rate during the transition from stillness to movement. We then compared the values of these parameters during the early and late trials of each session. To quantify changes in firing rate, we used the $n f d$ (the difference in firing rates during the early and late trials, divided by their sum; see Materials and Methods). This allowed us to measure the extent to which each neuron modified its activity during movement from the beginning of a session to its end. We found that the means of these distributions were statistically similar during the first $3 \mathrm{~d}$ (average $n f d, 0.04,0.02$, and $0.02 ; n=172,214$, and 202 on days $1-3$, respectively; ANOVA, $p>0.3$; each neuron contributed independently to both left and right movements). The fact that the average $n f d$ was near 0 implies that there was a balance between neurons that increased or decreased their firing rate over the course of each day. The variance of the $n f d$ function measures the extent to which the firing rate changed during a session. A larger variance indicates a more pronounced average change in firing rate. The $n f d$ variance on the first day was significantly larger than the variance calculated on the following days ( $F$ test, $p<0.01)$. This suggests that, during the first day of training, MI neurons gradually and selectively changed their firing rate during movement. These changes in firing rates did not lead to a change in the mean firing rate of the neuronal ensemble over days $(n=172,214$, and 202 on days $1-3$, respectively; ANOVA, $p>0.95$ ), indicating that the execution of faster movements was not achieved by increasing the ensemble average firing rate.

To quantify the changes in neuronal firing in relation to movement initiation, we counted trials in which the firing rate changed significantly (Pauluis and Baker, 2000) within a time window of $\pm 200 \mathrm{msec}$ around movement initiation. The number of trials in which the neuronal firing rate was modulated increased significantly during day 1 (Fig. $4 C$; from $43 \pm 2$ to $55 \pm$ $2 \%$; paired $t$ test, $p<0.001)$. On the second and third days of training, the percent of trials showing modulation in firing did not change (Fig. $4 C ; 56 \pm 2 \%$ in each session; $p>0.1$ ). This result implies that the depth of firing modulation between reaction time and movement time and consistency of the firing in relation to movement initiation increased with learning. The percent of 


\section{First day}

A
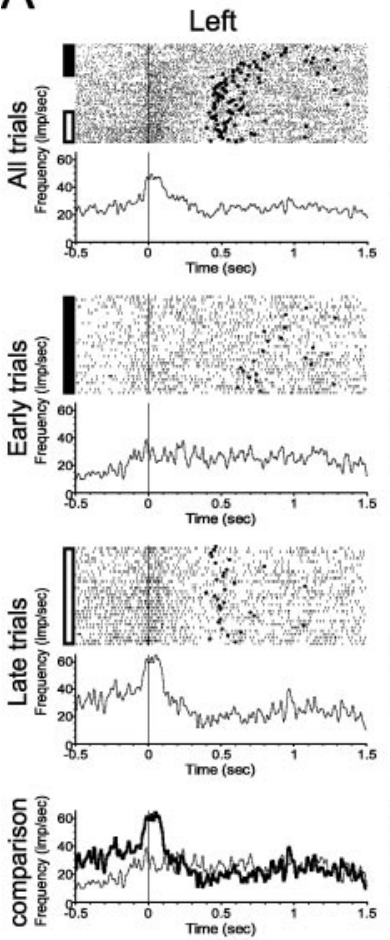

C
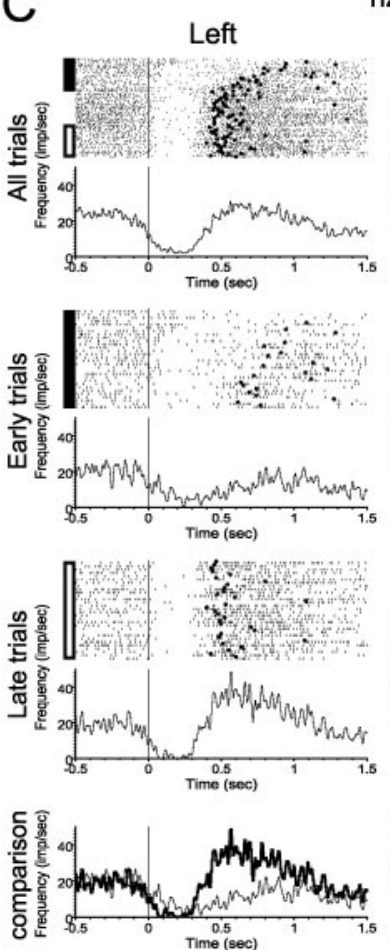

n24
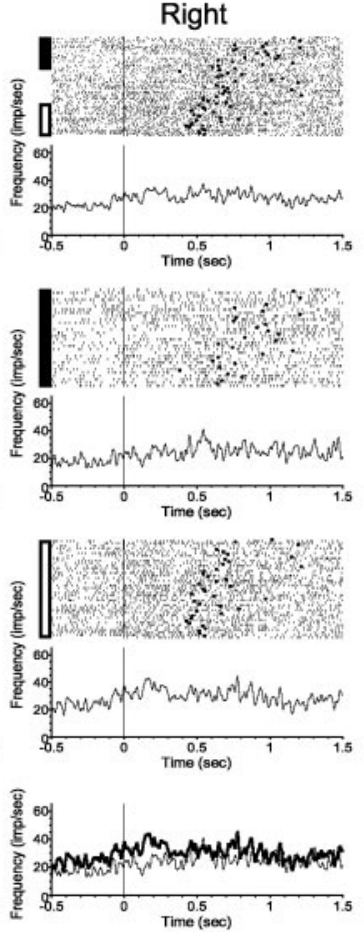

n28
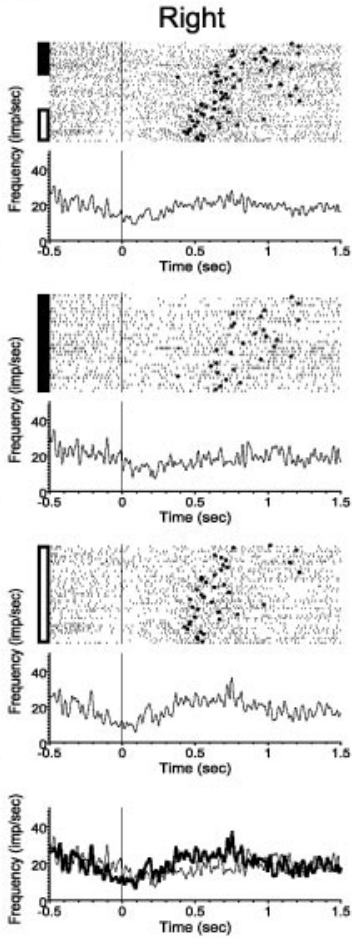

B

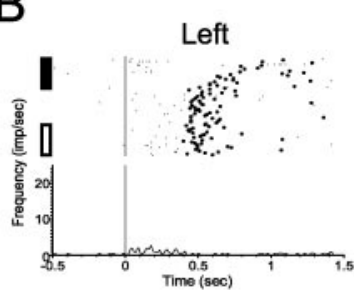

n08
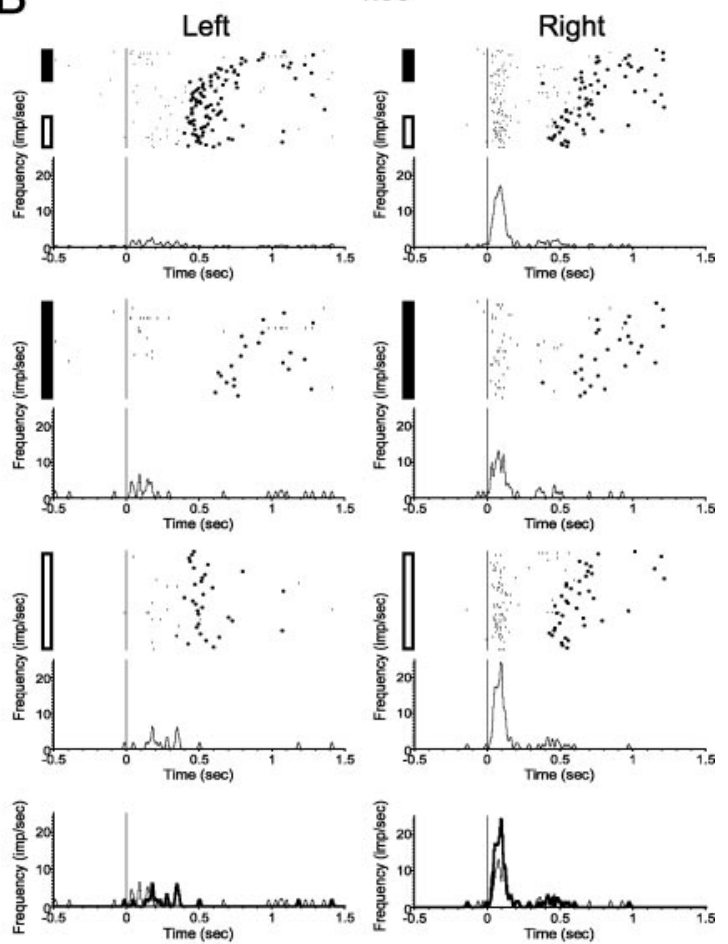

D

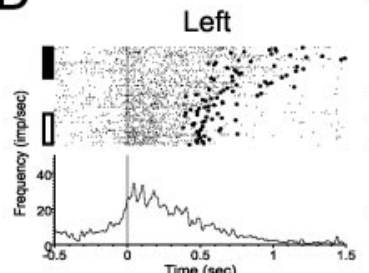

$\mathrm{n} 112$
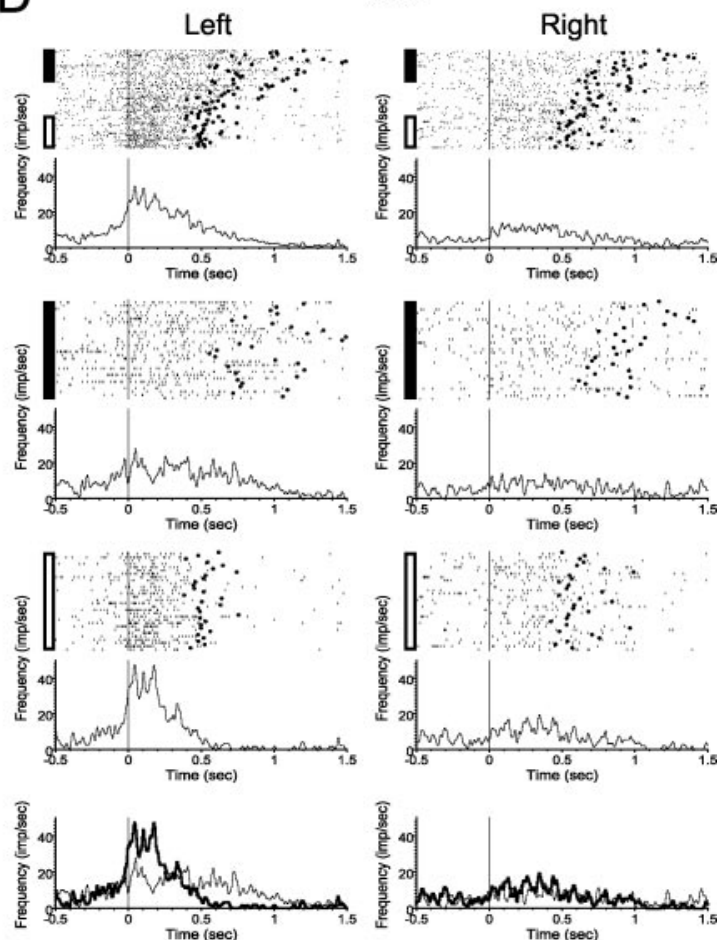

Figure 2. On day $1, \mathrm{MI}$ neurons exhibited a variety of changes in firing patterns during execution of the task. $A-D$, Each panel of graphs shows activity from one neuron. Each graph shows raster plots and the corresponding PETHs, aligned at the beginning of each movement (marked by a black line). The end of each movement is marked by a filled circle. In each graph, trials are presented top to bottom as they were presented during training. The left column in each panel shows activity during left movements, and the right column shows the activity during right movements. The top two graphs show the activity of each neuron observed over the course of the day. The second and third rows show the activity during the early and late trials, respectively. The bottom two graphs show the superimposed PETHs during the early (thin line) and late (thick line) trials. $A$, Firing modulation developed to one or both directions (in this cell, only to the left). $B$, The firing pattern became more pronounced. C, A decrease in firing developed or strengthened. D, The duration of movement-related activity was reduced. 


\section{Second day}

A
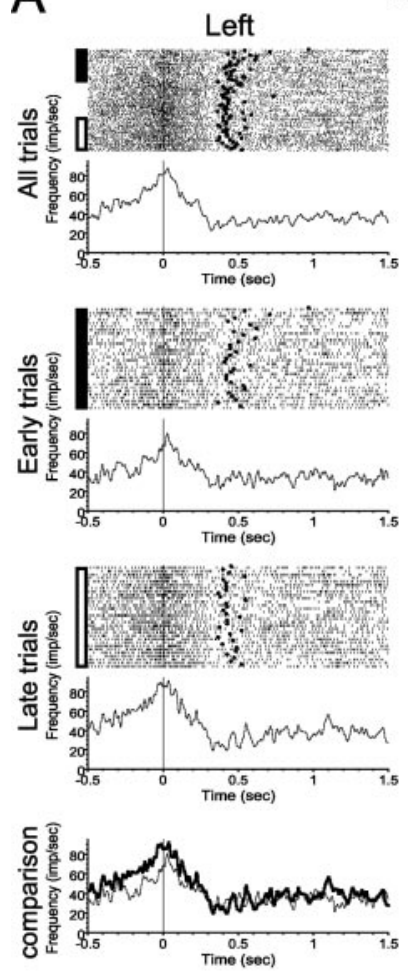

C
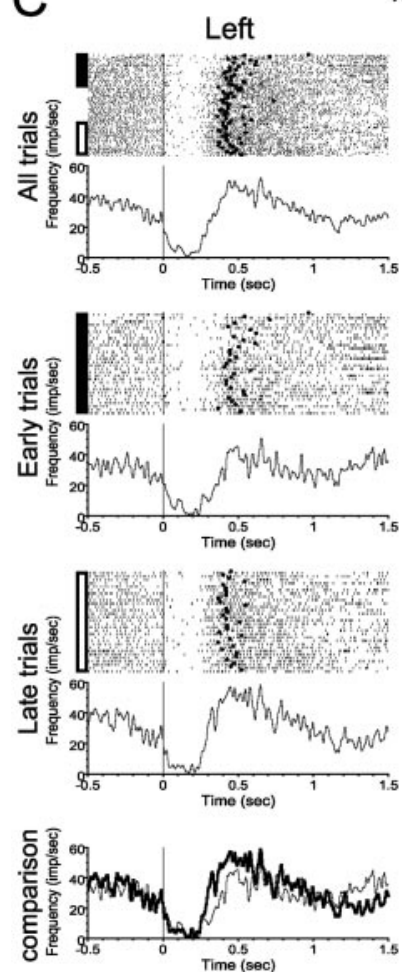

n24
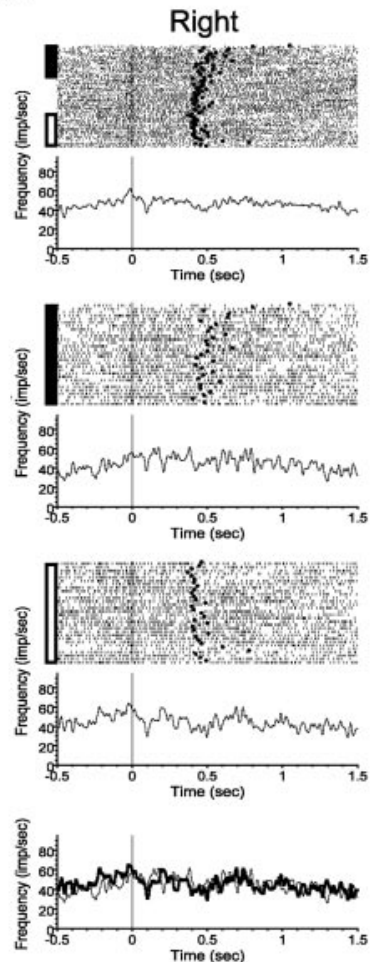

n28
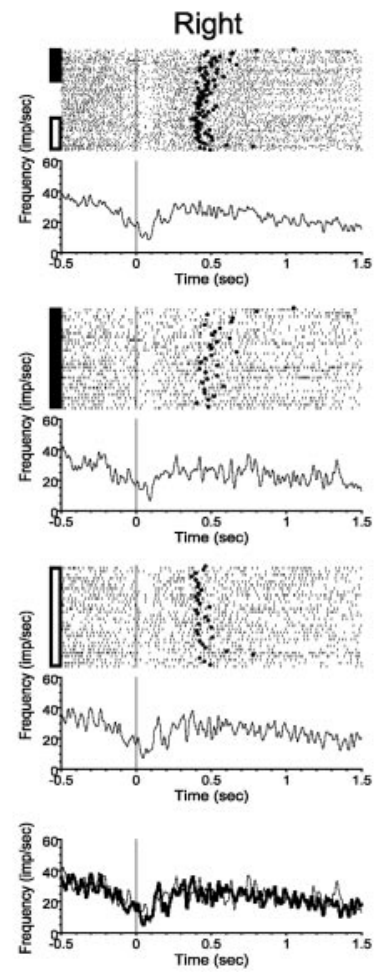

B

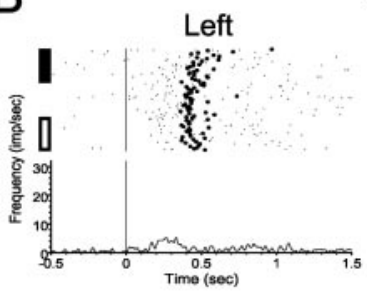

n08
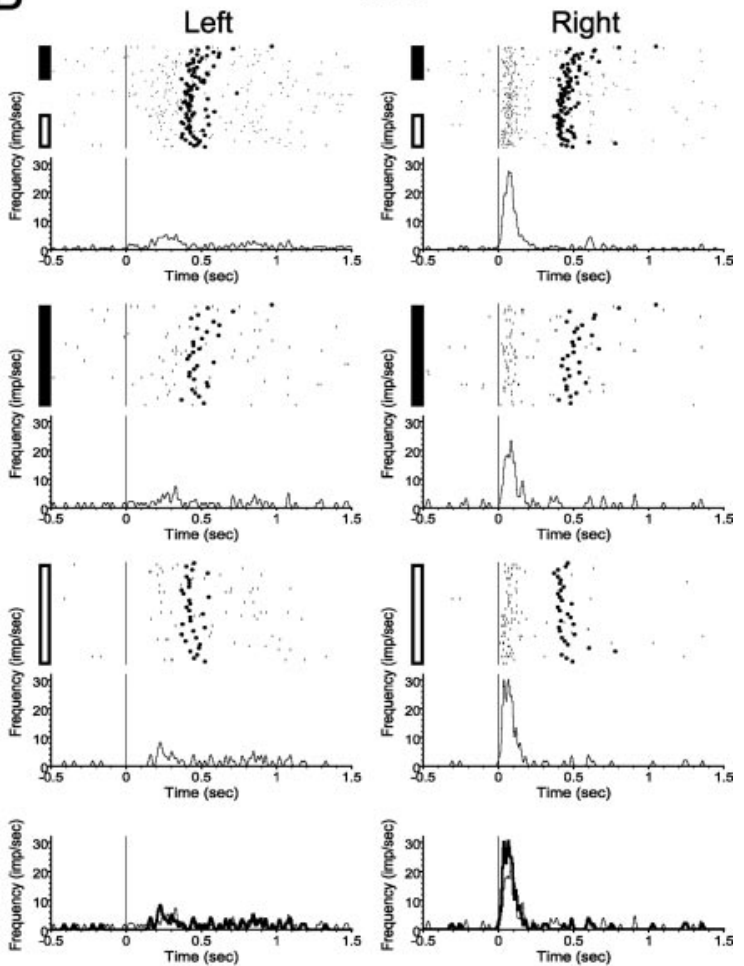

D

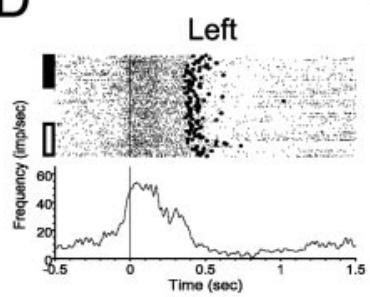

$\mathrm{n} 112$
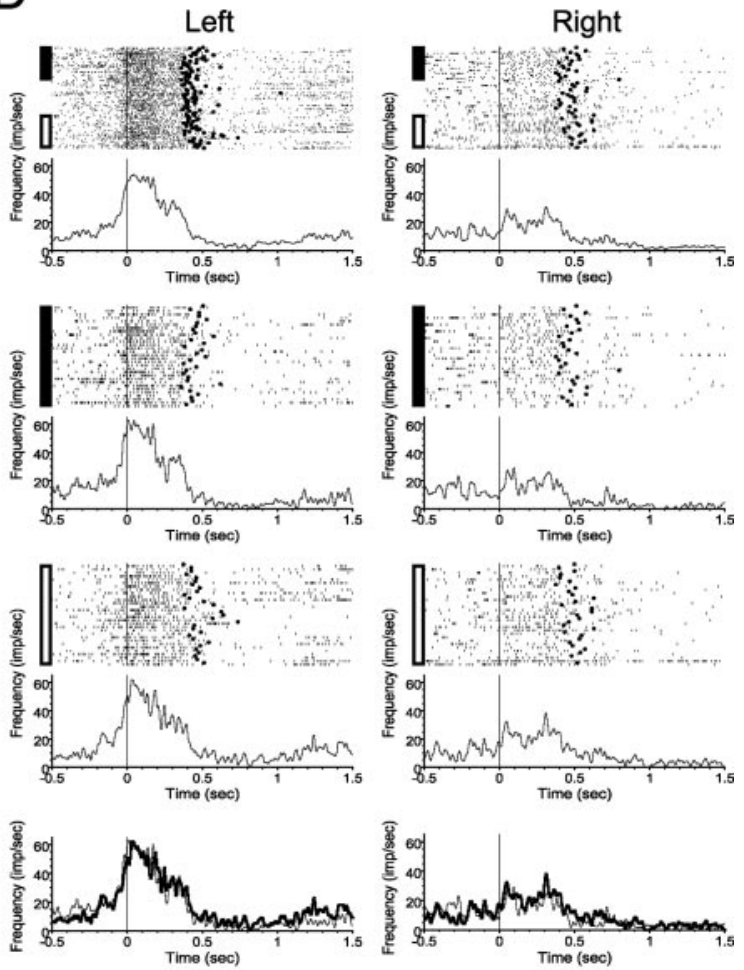

Figure 3. Neural activity was relatively stable over the course of the second and third days. As in Figure 2, each panel shows activity from a single neuron, this time for day 2. Spike sorting indicated that these four cells were probably the same as the examples used in Figure 2, and the firing patterns of these neurons on day 2 are similar to their activity at the end of day 1. All panels are as in Figure 2. 
A
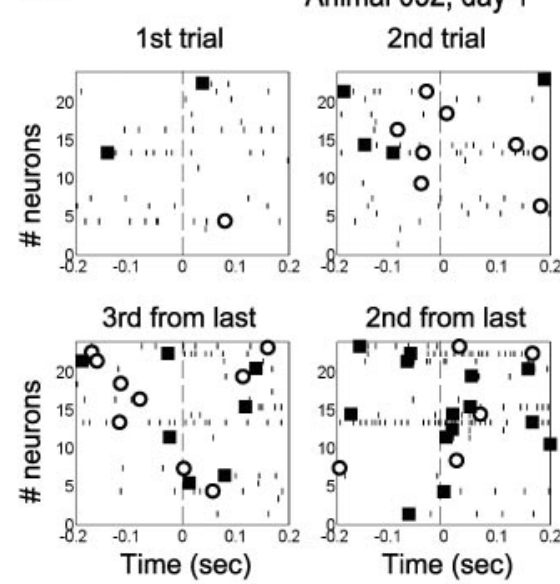

Animal 032 , day 1
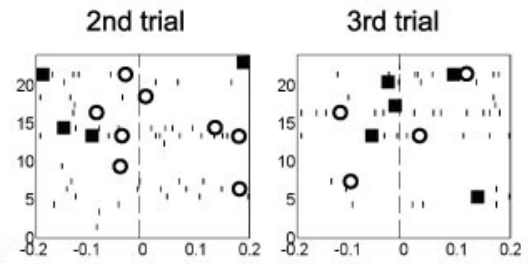

B

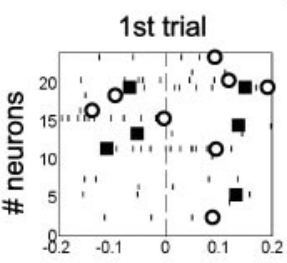

Animal 032 , day 2
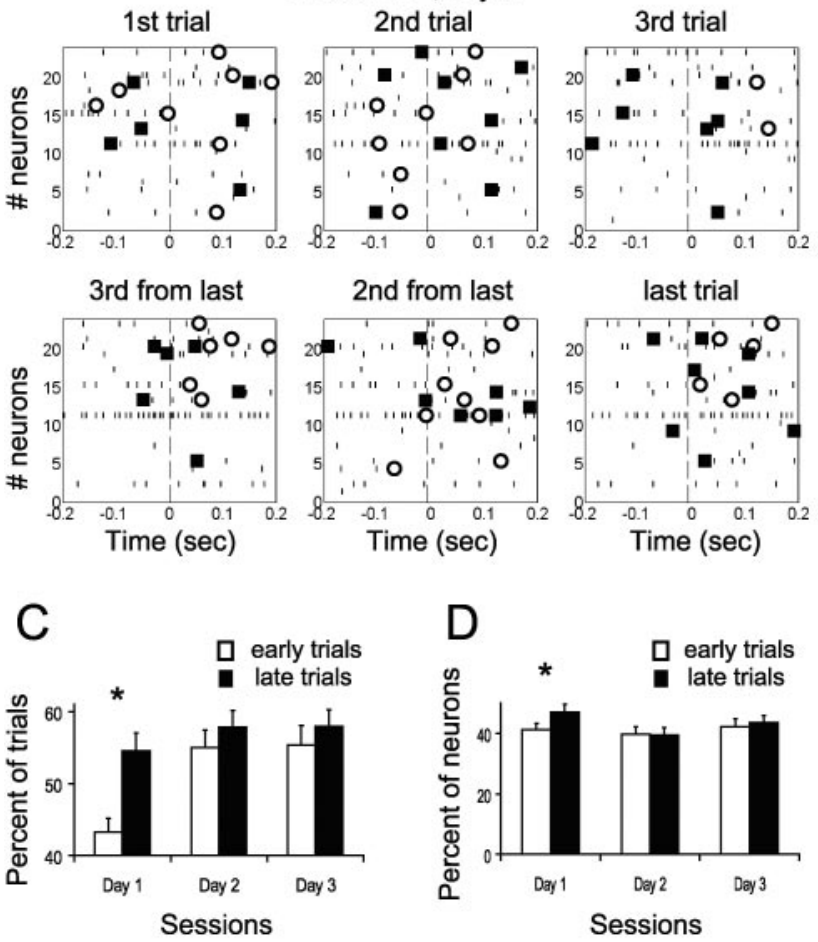

Figure 4. Number of neurons that changed their firing rate around movement initiation increased with practice. $A, B$, The raster plots show activity of all the neurons recorded simultaneously in one animal during the first three trials and the last three trials to the left for the first $(A)$ and second $(B)$ days. A significant increase in firing rate is marked by a filled square, and a significant decrease is marked by an open circle. Trials were aligned to movement initiation (dashed line). On the first day, the number of neurons with modified firing increased from 6 during the initial three trials to 13.7 during the last three trials. On the second day, the number of neurons with modified firing was 9.7 and 9 during the first and last three trials, respectively. C, The bar plot shows the percent of trials during which MI neurons significantly increased their firing rate near movement initiation for the early and late trials of days 1-3. A significant increase occurred during the first day ( $n=172,214$, and 202 on days 1-3, respectively; each neuron contributed independently to left and right movements). $D$, The average of the number of neurons per trial that significantly modified firing near movement initiation is shown for days $1-3$. The number of neurons per trial showing activity modulation significantly increased only during the first day $(n=6)$.

trials showing activity modulation around the time at which rats nose poked the center hole (trial initiation) did not change over days ( $54 \pm 11,53 \pm 10 \%$, and $51 \pm 11 \% ; p>0.5)$. This suggests that the increase in firing modulation and consistency of MI neurons is a selective process that occurs only during the acquisition of unfamiliar movements (i.e., left and right movements) and not during the execution of familiar movements (i.e., nose poking the center hole).

The increase in firing modulation of single neurons also implies that the number of neurons showing significant activity modulation per single trial increased over the course of day 1 (see example in Fig. 4A) but not in the following days (Fig. 4B). To quantify the behavior of the neuronal population near movement initiation, we calculated, for each trial, the number of MI neurons that modified their firing within a window of $200 \mathrm{msec}$ before and after movement initiation and averaged over the early and late trials separately. As expected, over animals, the number of neurons that exhibited significant modulations (either increase or decrease) in firing rate increased significantly during the first day ( $41 \%$ during the early trials to $47 \%$ during the late trials) but not on the following days ( 40 and $43 \%$ on the second and third days, respectively; Fig. $4 D$; ANOVA, $p<0.05$ ). The time course of the increase in the number of neurons with modified firing seems similar to the time course of the decrease in movement duration shown in Figure $1 D$. This suggests that synchronized neuronal firing rate modulations in MI may be important to generating coordinated motor command, thus allowing the animal to execute rapid movement.

\section{The discriminability of neuronal activity}

The discriminability of MI neuronal firing during movement to opposite directions is expected to increase with practice (i.e., as movements toward each side become more directed). To test this prediction, we compared both the mean and variance of individual spike count histograms for leftward and rightward movements that were executed early and late in each session (Fig. 5). These movement time histograms (MTHs) were calculated using a fixed time window of $250 \mathrm{msec}$ starting at movement initiation. During the first session, the MTHs calculated for left and right movements shifted differentially (Fig. 5B,C). The mean of each histogram during movement to one side could increase, decrease, or remain unchanged. Namely, changes in the histogram calculated for one direction showed no obvious relationship to changes that occurred in the histogram of the opposite direction. Over the neuronal population, the average difference in the mean of the histograms to the left and right increased during the first day (Fig. $5 D ; 0.7 \pm 0.1$ to $1.1 \pm 0.1$ ) but not on the following days $(0.9 \pm 0.1$ to $1 \pm 0.1$ and $1.0 \pm 0.1$ to $1.1 \pm 0.1$, on days 2 and 3 , respectively). The increase in the MTHs mean difference was significant on the first day (ANOVA, $p<0.01$, post hoc analysis). This result shows that the discriminability of MI neurons between left and right movements improved with practice.

Next, we tested whether the precision of the neural firing increased. To do that, we compared the mean and variance of the MTHs to both movement directions during the early and late trials of each session. Despite the changes in neural activity described thus far, the ratio between the mean and variance of the MTHs did not change during the first day (Fig. 6A); that is, although we expected to see the variance decrease over the course of the day, we found that when the neuronal firing rate increased, the variance increased with it. This result shows that learning to execute directed and precise movements had no apparent affect on the precision with which single neurons fired. Thus, the improvement in discriminability achieved by an increase in firing rate during learning was counteracted by the reduction in precision that resulted from a proportional increase in firing variability. Hypothetically, if the improvement in discriminability is 

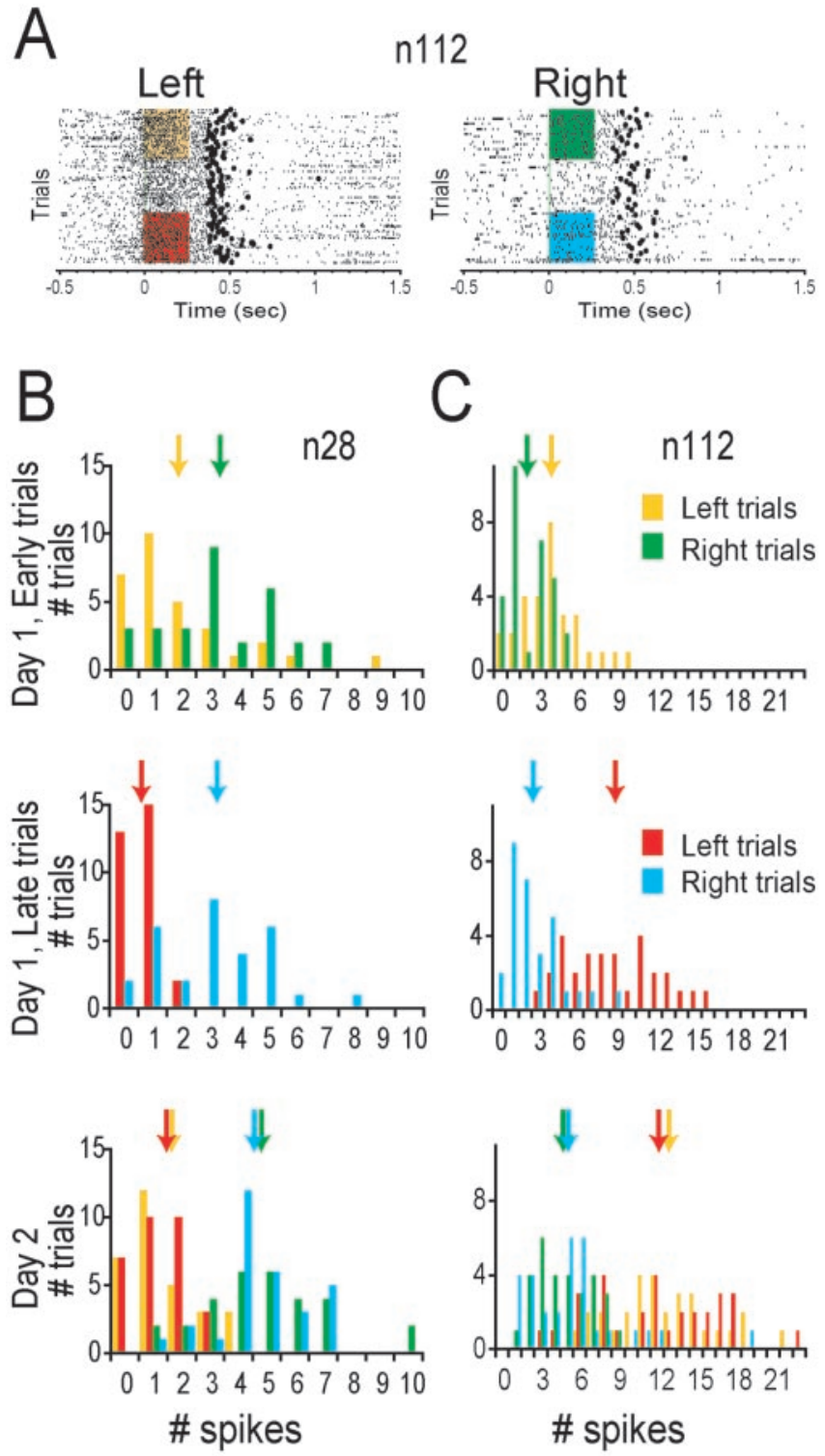

D

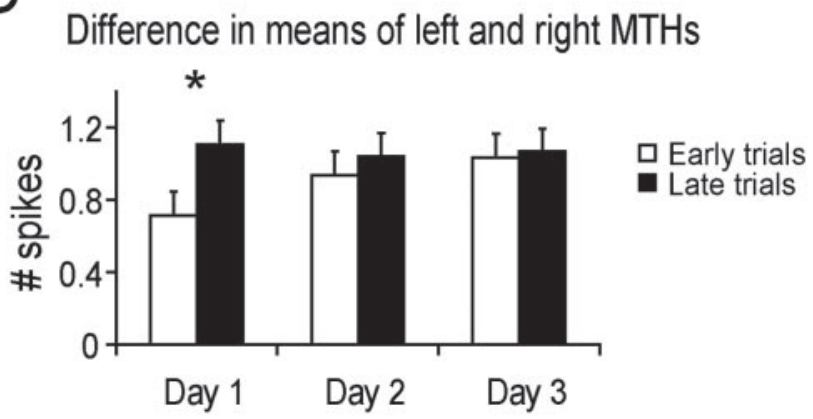

Figure 5. Discriminability of left and right movements based on spike count histograms increased with training. $A$, Raster plots of movements to the left and right for the same neuron. The color boxes provide the color code for the MTHs of the early and late trials used in $B$ and $C . B$, Spike counts of a single neuron were averaged over an epoch from 0 to 250 msec after movement initiation. Averages (arrows) are marked both for the early and late trials of the first day (top 2 rows) and on the second day (bottom row). C, Same as B for a different neuron. D, Histogram of the difference in the means of the MTHs to the left and right over the whole population shown for the early and late trials of days $1-3$. This difference in activity between left and right increased significantly during the first day but not during subsequent days ( $n=86$, 107 , and 101 on days $1-3$, respectively). large enough, it could result in a complete separation of the left and right MTHs despite the increase in variability. This would imply that downstream elements of the motor system (such as spinal cord) would be obliged to learn to integrate differently high and low firing rates to yield gradual and precise muscle activation. Here, none of MI neurons consistently provided reliable information about movement direction in all trials.

On days 2 and 3 , the regression line fit to the mean variance plot had a somewhat smaller slope than the regression line of day 1 (Fig. 6B). However, this change is small and probably arises from the fact that the firing rate was changing during day 1 , even during the 30 trials of the early and late periods. The changing firing rate would result in an apparent increase of the variance.

\section{The ensemble}

To evaluate whether the uncertainty in movement direction introduced by single neurons was reduced at the level of the neural ensemble, we developed an algorithm that estimates the statistical similarity of neural activity over different trials (see Materials and Methods). As expected given our findings regarding the relationship of mean and variance, the best prediction of movement direction by single neurons (Fig. $6 \mathrm{C}$, open circles) did not improve with training $(79,84$, and $82 \%$ on days $1-3$, respectively; ANOVA, $p>0.5$ ), nor did the average prediction of single neurons (Fig. $6 C$, open triangles; 63, 63, and 64\%; ANOVA, $p>0.9$ ). Furthermore, the distribution of prediction of movement direction by single neurons did not change over days (i.e., with training, prediction of movement direction by some neurons improved and by others became worse; Fig. $6 E$ ). In contrast, the prediction of movement direction by the ensemble improved significantly with training $(72,92$, and $89 \%$ on days $1-3$, respectively; Fig. $6 C$, rectangles; paired $t$ test, $p<0.005$; Fig. $6 D$; ANOVA, $p<0.01$ ). We used the waiting period as a control for the performance of the algorithm on changes from day 1 to day 3 that might not have been task-relevant. During the waiting period, the rats could not know which direction would be required, and the data should not contain any information about movement direction. Indeed, predictions of movement direction of the neuronal ensemble were at a chance level (56, 57, and 53\%; Fig. $6 D$ ). These results suggest that, with learning, the neuronal ensemble may be able to overcome the uncertainty introduced by the variability in firing of single neurons.

\section{Discussion}

Performance improves during motor skill learning. In the present study, we measured a significant reduction in movement duration on the first training day. Such a reduction can occur when rats perform a shorter trajectory, increase their movement velocity, or both. In either case, the movements executed early in training are different than those executed late in training. Despite the fact that, before each session, the animals are water-deprived for $>20 \mathrm{hr}$, before training they are not moving as fast as after training. In addition, as movements become faster, the precision with which the rats move increases. This is reflected in reduction of movement time variability. What neural mechanisms might explain this improvement in movement execution? We observed changes in the activity of single MI neurons that had a time course similar to the reduction in movement time. First, significant changes in firing rate during movement occurred only when the decrease in movement duration was significant (i.e., day 1), and, second, as learning progressed, more neurons rapidly changed their firing rate near movement initiation, which may contribute to faster movement onset. Previous studies of motor learning 

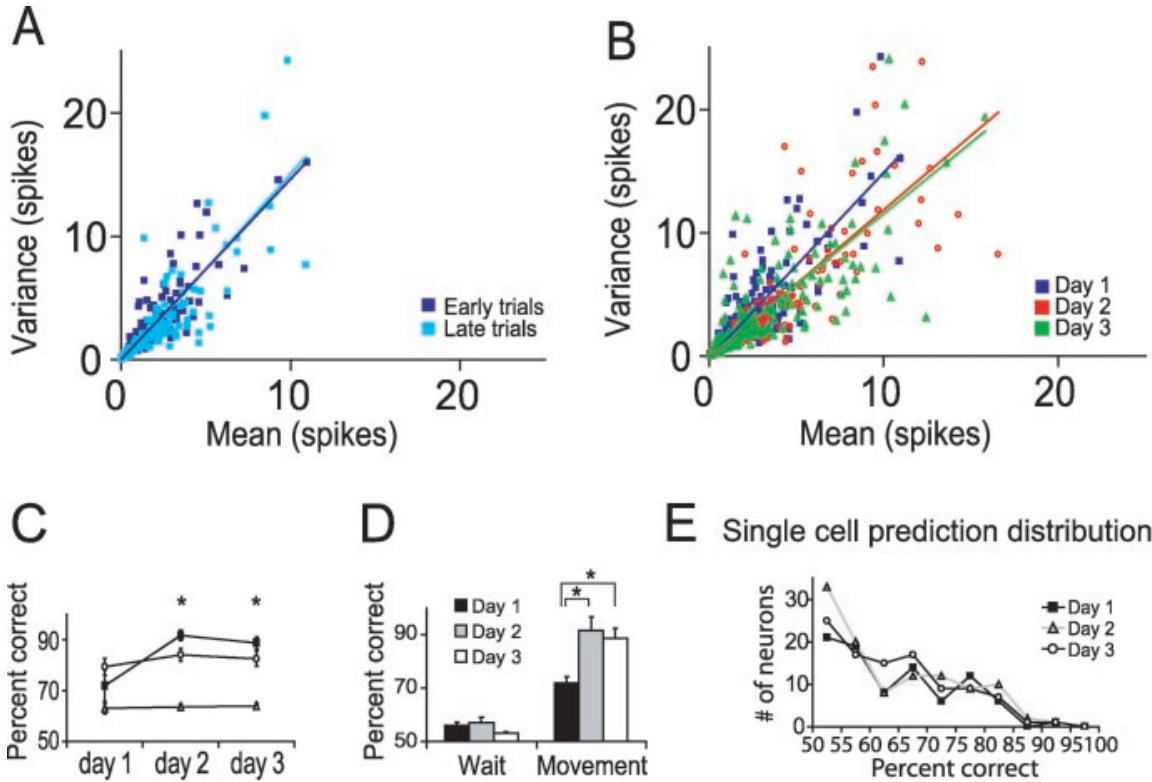

Figure 6. Movement direction was better predicted by the ensemble compared with single neurons. The ratio between the mean firing and the variance in firing of individual MI neurons did not change with learning. $A$, The variance of the MTHs was plotted against their means for all neurons during movements to the left and right during the early (dark blue) and late (light blue) trials of the first day. The regression line fit to the plot of the early trials was similar to that of the late trials ( $n=172$; each neuron contributed to movement both directions). $B$, Variance-mean plots are shown for all days. The slopes of the regression lines were reasonably approximated by a Poisson distribution in which the variance equals the mean $(A, B$, black lines; $n=172,214$, and 202 on days $1-3$, respectively). $C$, Different lines show the best predictions of movement direction by single neurons (open circles), the average predictions of single neurons (open triangles), and the predictions of movement direction by the ensemble (filled squares) for $3 \mathrm{~d}$ of training. Predictions of movement direction by the ensemble became significantly better than the best prediction of single neurons $(n=6)$. D , Histograms of the predictions of movement direction by the ensemble during the waiting period and during movement for the $3 \mathrm{~d}$ of training. Prediction of the ensemble significantly improved with training $(n=6)$. $E$, Distributions of the predictions of a single neuron for days $1-3$. These distributions did not change with learning.

have shown changes in the average firing rate and shifts in the preferred direction during primate reaching movements (Mitz et al., 1991; Wise et al., 1998; Gandolfo et al., 2000; Li et al., 2001; Paz et al., 2003). Here, because the animals move in two directions, it is difficult to evaluate whether the observed changes in firing rate reflect shifts in the preferred direction or changes in the average activity. It may be possible to distinguish between the two options by characterizing movement kinematics.

Could the observed changes in single-neuron activity also account for the reduction in movement variability? On one hand, the discriminability of neural activity during left and right movements improved, as expected from performance of more direct movements. However, translating the increased discriminability in single-neuron firing into movement precision is not trivial. On the other hand, the variability in single-neuron firing did not decrease with the reduction in movement variability. Moreover, when the firing rate of single neurons increased, their precision decreased because of a proportional increase in firing variability. Thus, it seems that learning-induced reduction in movement variability may reflect changes in the neuronal ensemble and not in single neurons. It has been reported that tuning width did not change during adaptation to an external force field ( $\mathrm{Li}$ et al., 2001). However, tuning width was calculated only after the behavior stabilized (i.e., the correlation coefficient of movements was high), and changes in tuning width do not necessarily correlate with firing variability during movement toward the same direction.

Our results demonstrate that the uncertainty in movement direction introduced by single-neuron variability is significantly reduced at the level of the neuronal ensemble (i.e., the ensemble activity in different trials becomes more similar when movements are performed in the same direction compared with single-neuron activity). This increased similarity in ensemble activity can potentially explain the reduced variability in the behavior. The ability of neuronal ensembles to reduce the effect of single-neuron variability could be accomplished in several ways. One plausible explanation would be to hypothesize that MI ensembles rely on a probabilistic model in which a motor command can be generated by any subgroup of task-defined neurons in which each cell fires randomly according to its time-dependent firing distributions. According to this hypothesis, when MI acquires a new movement, the firing distributions of single neurons change until their time-dependent averaged activity reaches a stable point from which further improvement would be inefficient. This model is corroborated by a theoretical study showing how small networks of $50-$ 100 neurons can reduce neuronal variability when presented with time-varying input (Shadlen and Newsome, 1998). Another theoretical study has suggested that the motor system may reduce variability in the task-relevant dimensions at the expense of the task-irrelevant dimensions (Todorov and Jordan, 2002). In this case, the ensemble may actively reduce noise in the direction of the learned movements (i.e., left and right), for example, by learning to generate neuronal noise correlations (Lee et al., 1998). This model implies that movements in other directions that were not optimized because of learning would not exhibit noise correlations. The sustained variability in the behavior of the animal, after performance reached a plateau, could stem from the inability of the ensemble to remove all of the variability introduced by individual neurons.

In primate reaching movements, joint angular velocity and the MI firing rate are correlated (Reina et al., 2001). Our findings generalize these results to the rat in which MI neurons that increased firing during movement further augmented their firing when the movement speed increased (measured as decreased movement duration). However, many MI neurons decreased firing during movement (Wise et al., 1998). These neurons decreased firing even more with the reduction in movement duration. All these changes in firing rate modulations were balanced so that the ensemble firing rate did not change during learning. Although it is possible that a sampling bias may have influenced the average changes in firing rate, increases and decreases in firing balanced rather precisely. This suggests that a mean firing rate may be kept constant across the MI population. Thus, our results provide experimental evidence consistent with the theoretical claim that a balance between inhibition and excitation should emerge naturally in certain large networks of excitatory and inhibitory neurons (van Vreeswijk and Sompolinsky, 1996).

Electrophysiological studies of associative learning have reported that the changes in neuronal activity during the reaction time lag the changes in performance of the animal (Mitz et al., 
1991; Wise et al., 1998; Paz et al., 2003). In all of these studies, the executed movement did not change. Here, we found that, during motor skill learning, the changes in neural activity develop in parallel with the behavioral changes. We did not find any measure of neural activity during movement that changed significantly on the second or third training days. Although one set of results is in primates, and our results are in the rat, this difference suggests a hypothesis whereby two separate processes that are involved in the improvement in task performance may exist. The first process would involve formation of an internal model of the executed movements, and the second would be the development of a transformation between sensory cues and movements. This hypothesis implies that the neural correlates of the tone-movement association would be pronounced after $>3 \mathrm{~d}$ because then behavior improved (i.e., the error rate decreased). Therefore, in our hands, it seems that an internal model of movement execution (motor skill learning) evolved before the internal model of sensorimotor transformation (associative learning), which is expected to have a stronger effect on activity during movement preparation. Thus, in the confines of the task used in the present study, it seems that formation of an internal model of movement is likely to have preceded the formation of a sensorimotor transformation in MI.

In conclusion, our results demonstrate that, during motor skill learning, individual neurons modify their average firing rate and their temporal pattern of activity. Learning to execute fast, directed movements does not affect an essential parameter of neuronal firing; firing rate variance continues to be proportional to the mean firing rate despite the behavioral changes. Therefore, although the learning-dependent changes in single-neuron firing may account for the reduction in movement duration, they cannot account for the reduction in movement variability. In contrast, prediction of movement direction by the neuronal ensemble improved significantly when calculated on the basis of similarity in the ensemble activity, suggesting that the ensemble is able to reduce the effect of firing variability of individual neurons. We propose that, with practice, neuronal ensembles learn to overcome the uncertainty introduced by the stochasticity of individual neurons and thus improve the animal's motor performance. As described above, several mechanisms may allow uncertainty reduction by the ensemble. Further study is required to distinguish between these plausible mechanisms, some of which may be also essential for other types of learning.

\section{References}

Averbeck BB, Lee D (2003) Neural noise and movement-related codes in the macaque supplementary motor area. J Neurosci 23:7630-7641.

Bonato C, Zanette G, Fiaschi A, Rossini PM (2002) Activity-dependent modulation of synaptic transmission in the intact human motor cortex revealed with transcranial magnetic stimulation. Cereb Cortex 12:1057-1062.

Dayan P, Abbott L (2001) Theoretical neuroscience: computational and mathematical modeling of neural systems. Cambridge, MA: MIT.

Dosher BA, Lu ZL (1998) Perceptual learning reflects external noise filtering and internal noise reduction through channel reweighting. Proc Natl Acad Sci USA 95:13988-13993.

Dubnov S, El-Yaniv R, Gdalyahu Y, Schneidman E, Tishby N, Yona G (2002) A new nonparametric clustering algorithm based on iterative estimation of distance profiles. Machine Learn 47:35-61.

Gandolfo F, Li C, Benda BJ, Schioppa CP, Bizzi E (2000) Cortical correlates of learning in monkeys adapting to a new dynamical environment. Proc Natl Acad Sci USA 97:2259-2263.
Georgopoulos AP, Schwartz AB, Kettner RE (1986) Neuronal population coding of movement direction. Science 233:1416-1419.

Germain L, Lamarre Y (1993) Neuronal activity in the motor and premotor cortices before and after learning the associations between auditory stimuli and motor responses. Brain Res 611:175-179.

Gold J, Bennett PJ, Sekuler AB (1999) Signal but not noise changes with perceptual learning. Nature 402:176-178.

Kleim JA, Barbay S, Nudo RJ (1998) Functional reorganization of the rat motor cortex following motor skill learning. J Neurophysiol 80:3321-3325.

Kleim JA, Barbay S, Cooper NR, Hogg TM, Reidel CN, Remple MS, Nudo RJ (2002) Motor learning-dependent synaptogenesis is localized to functionally reorganized motor cortex. Neurobiol Learn Mem 77:63-77.

Laubach M, Wessberg J, Nicolelis MA (2000) Cortical ensemble activity increasingly predicts behaviour outcomes during learning of a motor task. Nature 405:567-571.

Lee D, Port NL, Kruse W, Georgopoulos AP (1998) Variability and correlated noise in the discharge of neurons in motor and parietal areas of the primate cortex. J Neurosci 18:1161-1170.

Li CS, Padoa-Schioppa C, Bizzi E (2001) Neuronal correlates of motor performance and motor learning in the primary motor cortex of monkeys adapting to an external force field. Neuron 30:593-607.

Lotze M, Braun C, Birbaumer N, Anders S, Cohen LG (2003) Motor learning elicited by voluntary drive. Brain 126:866-872.

Mitz AR, Godschalk M, Wise SP (1991) Learning-dependent neuronal activity in the premotor cortex: activity during the acquisition of conditional motor associations. J Neurosci 11:1855-1872.

Muellbacher W, Ziemann U, Boroojerdi B, Cohen L, Hallett M (2001) Role of the human motor cortex in rapid motor learning. Exp Brain Res 136:431-438.

Muellbacher W, Ziemann U, Wissel J, Dang N, Kofler M, Facchini S, Boroojerdi B, Poewe W, Hallett M (2002) Early consolidation in human primary motor cortex. Nature 415:640-644.

Nicolelis MA, Ghazanfar AA, Faggin BM, Votaw S, Oliveira LM (1997) Reconstructing the engram: simultaneous, multisite, many single neuron recordings. Neuron 18:529-537.

Nicolelis MA, Dimitrov D, Carmena JM, Crist R, Lehew G, Kralik JD, Wise SP (2003) Chronic, multisite, multielectrode recordings in macaque monkeys. Proc Natl Acad Sci USA 100:11041-11046.

Pauluis Q, Baker SN (2000) An accurate measure of the instantaneous discharge probability, with application to unitary joint-even analysis. Neural Comput 12:647-669.

Paz R, Boraud T, Natan C, Bergman H, Vaadia E (2003) Preparatory activity in motor cortex reflects learning of local visuomotor skills. Nat Neurosci 6:882-890.

Poggio T, Fahle M, Edelman S (1992) Fast perceptual learning in visual hyperacuity. Science 256:1018-1021.

Reina GA, Moran DW, Schwartz AB (2001) On the relationship between joint angular velocity and motor cortical discharge during reaching. J Neurophysiol 85:2576-2589.

Remple MS, Bruneau RM, VandenBerg PM, Goertzen C, Kleim JA (2001) Sensitivity of cortical movement representations to motor experience: evidence that skill learning but not strength training induces cortical reorganization. Behav Brain Res 123:133-141.

Rioult-Pedotti MS, Friedman D, Donoghue JP (2000) Learning-induced LTP in neocortex. Science 290:533-536.

Shadlen MN, Newsome WT (1998) The variable discharge of cortical neurons: implications for connectivity, computation, and information coding. J Neurosci 18:3870-3896.

Shadmehr R, Holcomb HH (1997) Neural correlates of motor memory consolidation. Science 277:821-825.

Todorov E, Jordan MI (2002) Optimal feedback control as a theory of motor coordination. Nat Neurosci 5:1226-1235.

Ungerleider LG, Doyon J, Karni A (2002) Imaging brain plasticity during motor skill learning. Neurobiol Learn Mem 78:553-564.

van Vreeswijk C, Sompolinsky H (1996) Chaos in neuronal networks with balanced excitatory and inhibitory activity. Science 274:1724-1726.

Wise SP, Moody SL, Blomstrom KJ, Mitz AR (1998) Changes in motor cortical activity during visuomotor adaptation. Exp Brain Res 121:285-299.

Wolpert DM, Ghahramani Z, Flanagan JR (2001) Perspectives and problems in motor learning. Trends Cogn Sci 5:487-494. 